\title{
Design and rationale of randomized CT-PRECISION study
}

\author{
Xu Dai ${ }^{1}$, Yarong $\mathbf{Y u}^{2}$, Lihua Yu ${ }^{1}$, Lei Zhang ${ }^{2}$, Jiayin Zhang ${ }^{2}$ \\ ${ }^{1}$ Institute of Diagnostic and Interventional Radiology, Shanghai Jiao Tong University Affiliated Sixth People's Hospital, Shanghai, China; \\ ${ }^{2}$ Department of Radiology, Shanghai General Hospital, Shanghai Jiao Tong University School of Medicine, Shanghai, China \\ Correspondence to: Jiayin Zhang, MD; Lei Zhang, MD. Department of Radiology, Shanghai General Hospital, Shanghai Jiao Tong University School \\ of Medicine, \#86 Wujin Rd, Shanghai 200080, China. Email: andrewssmu@msn.com; zhanglei4302@hotmail.com.
}

Background: Coronary computed tomography angiography (CCTA) combined with dynamic CT
myocardial perfusion imaging (CT-MPI) and CCTA combined with CT fractional flow reserve (CT-FFR)
are both expected to be efficient one-stop shop imaging strategies to guide clinical management. The aim
of the study is to determine which of these two methods has superiority in terms of guiding treatment in
patients with intermediate to high pretest probability of coronary artery disease (CAD). Methods: CT-PRECISION (Computed Tomography myocardial PeRfusion imaging vErsus Computed tomography derived fractional flow reServe: impact $\mathrm{ON}$ guiding treatment and prognosis in patients with intermediate to high pretest probability of CAD) is a multicenter, prospective, open-label, randomized study to directly compare the clinical value of guiding treatment and prognostic discrimination of CCTA + dynamic CT-MPI strategy and CCTA + CT-FFR strategy in patients with intermediate to high pretest probability of CAD. Four hundred and twelve patients will be enrolled in this study and randomized to CCTA + dynamic CT-MPI arm and CCTA + CT-FFR arm. All patients will be followed up for at least 2 years. The primary endpoint is the rate of unnecessary invasive coronary angiography (ICA) within 90 days, which is defined as ICA without revascularization. The secondary endpoints will include: (I) a composite of major adverse cardiac events (MACE, defined as all-cause mortality, non-fatal myocardial infarction, rehospitalization due to aggravated angina symptoms, and late revascularization); (II) symptom change at 1 year; (III) the rate of late revascularization after CT examination; (IV) reclassification rate of CCTA + dynamic CT-MPI and CCTA + CT-FFR guided strategies compared with CCTA alone; (V) overall radiation dose, contrast media usage and medical cost.

Discussion: The study will provide valuable information about the optimal CT-based diagnostic strategy with regard to the clinical management of patients with intermediate to high pretest probability of CAD.

Trial registration: The study is registered at Chinese Clinical Trial Registry (ChiCTR) with the identifier number ChiCTR2000041102. The first enrollment is planned for January 2021.

Keywords: Coronary artery disease (CAD); fractional flow reserve (FFR); computed tomography angiography; myocardial perfusion imaging (MPI); major adverse cardiac events (MACEs)

Submitted Jan 27, 2021. Accepted for publication Mar 28, 2021.

doi: $10.21037 / \mathrm{cdt}-21-57$

View this article at: http://dx.doi.org/10.21037/cdt-21-57

\section{Introduction}

Coronary computed tomography angiography (CCTA) is a well-established non-invasive diagnostic modality for anatomic assessment of coronary stenosis but its clinical value is limited by low specificity for detecting lesionspecific ischemia. The PROMISE trial has demonstrated that CCTA increased the rate of invasive coronary angiography (ICA) by $50 \%$ as compared with functional test and over $1 / 4$ of these patients didn't have obstructive coronary artery disease (CAD) identified by ICA (1). Recently, the technical development of CT scanners and application of computational fluid dynamics model enables dynamic CT myocardial perfusion imaging (CT-MPI) 
and fractional flow reserve derived by CT angiography (CT-FFR) to provide functional information of coronary lesions based on CT data. The NXT trial showed that CTFFR can accurately predict lesion-specific ischemia with reference to invasive FFR, with a per-patient sensitivity and specificity of $86 \%$ and $79 \%$, respectively (2). Moreover, CT-FFR can reduce unnecessary ICA, as compared with routine CCTA $(3,4)$. In addition to CT-FFR, dynamic CTMPI is now feasible to provide quantitative myocardial perfusion parameters at relatively low radiation dose and contrast media usage using third generation of dual source CT. These parameters are able to precisely evaluate myocardial ischemia with reference to invasive FFR or cardiac MR MPI (4,5). A recent study has demonstrated that CCTA combined with dynamic CT-MPI (CCTA + dynamic CT-MPI) strategy can also reduce unnecessary ICA, as compared with CCTA strategy alone (6). Although CCTA + dynamic CT-MPI and CT-FFR have great potential in functional assessment of $\mathrm{CAD}$, these two novel imaging modalities also have the following pros and cons. CCTA + dynamic CT-MPI has better diagnosis accuracy, especially higher positive predictive value, than does CTFFR (7). However, the additional acquisition of CCTA + dynamic CT-MPI protocol results in higher radiation dose and contrast medium usage (8). CT-FFR simulation only needs routine CCTA dataset (9) but the diagnostic performance is limited in cases with impaired image quality, severe calcification and "grey zone" lesions (10).

Functional assessment is recommended in patients with intermediate-to-high pre-test probability of CAD in order to optimize treatment strategy (11). CCTA + dynamic CT-MPI and CCTA + CT-FFR are both expected to be efficient one-stop shop imaging strategies to guide clinical management. However, there is no existing data for direct comparison of these two protocols to determine which one has superiority for treatment guiding. Given the better diagnostic performance of dynamic CT-MPI, we hypothesized that CCTA + dynamic CT-MPI strategy may result in lower rate of unnecessary ICA and similar prognosis compared to CCTA + CT-FFR strategy in patients with intermediate to high pre-test probability of CAD. Therefore, this CT-PRECISION (Computed Tomography myocardial PeRfusion imaging vErsus Computed tomography derived fractional flow reServe: Impact $\mathrm{ON}$ guiding treatment and prognosis in patients with intermediate to high pretest probability of CAD) study is designed to investigate the rate of unnecessary ICA and clinical outcome in patients guided by CCTA + dynamic CT-MPI strategy and CCTA + CT-FFR strategy. We present the protocol in accordance with the SPIRIT reporting checklist (available at: http://dx.doi.org/10.21037/ cdt-21-57).

\section{Methods}

The study will be conducted in accordance with the Declaration of Helsinki (as revised in 2013). Hospital ethic committees of Shanghai General Hospital and Shanghai Jiao Tong University Affiliated Sixth People's Hospital approve this prospective study (approval ID: 2020-173) and all patients will be asked to sign informed consents before enrollment. Any amendments of the protocol will be submitted to hospital ethic committees for review. Detailed clinical characteristics will be recorded.

\section{Study population}

The study subjects will be enrolled from two tertiary medical centers. Competitive enrollment and random distribution methods will be adopted. Patients will be included if they: (I) have stable angina or atypical angina; (II) have intermediate to high pre-test probability $(\geq 15 \%)$ of CAD estimated by Updated Diamond-Forrester score; (III) are $\geq 18$ years old.

Patients will be excluded if they: (I) have unstable angina; (II) have previous history of myocardial infarction or coronary revascularization; (III) have severe cardiac arrhythmia; (IV) are suspected of cardiomyopathy or other diseases affecting myocardial perfusion; (V) have renal insufficiency; (VI) have allergic reaction to iodinated contrast medium; (VII) have contraindications to the usage of adenosine triphosphate (ATP) (such as asthma, hypotension, III atrioventricular block).

\section{Randomization procedure}

Patients will be randomized at 1:1 ratio (using computed generated random numbers) to CCTA + dynamic CT-MPI group or CCTA + CT-FFR group and the downstream treatment strategy will be decided by referring cardiologists according to the CT-based imaging findings. Simple randomization will be used in this research, aiming to achieve a 1:1 allocation ratio. Randomization sequence will be independently generated through software (SPSS 22.0) by a statistician who will not participate rest of the research. Random seed will be determined according to time of 


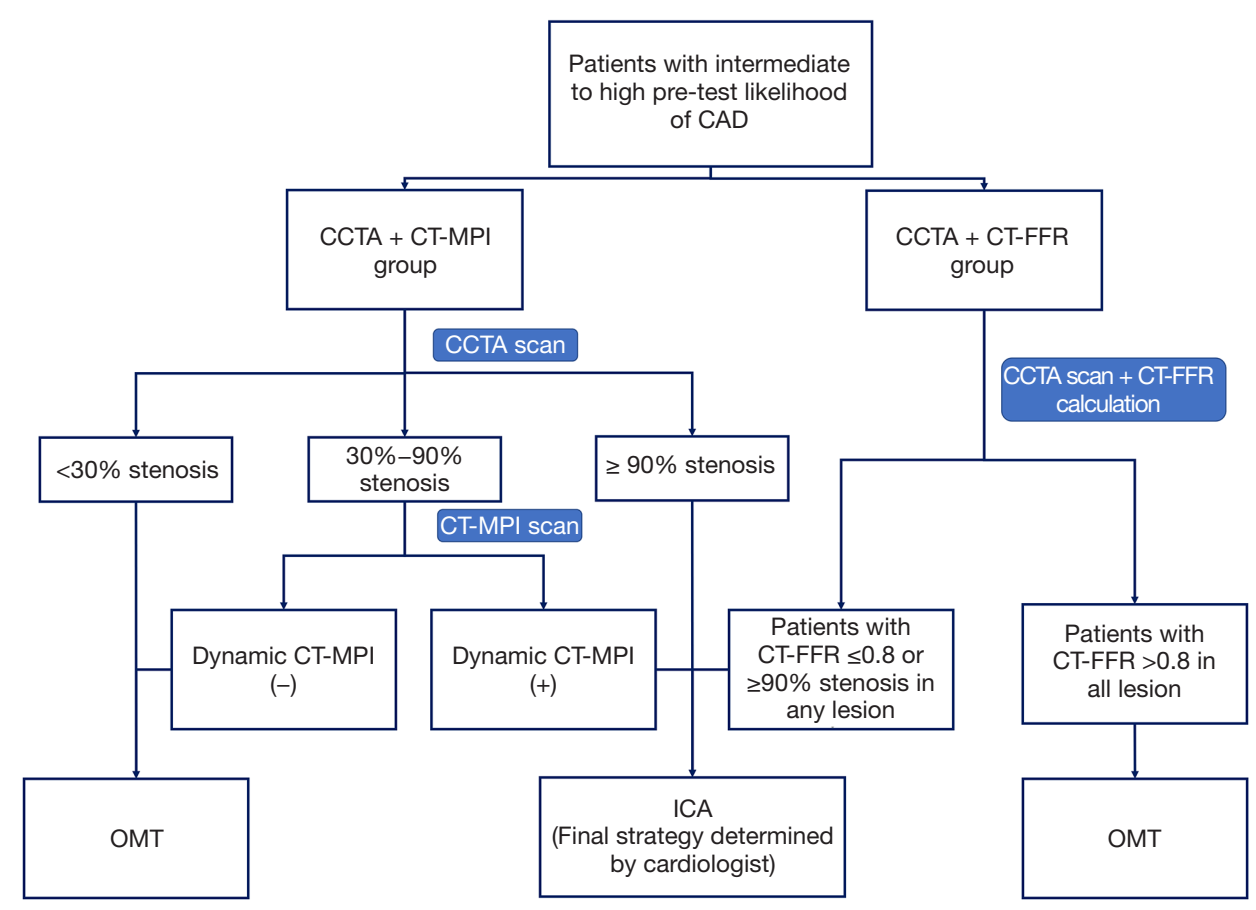

Figure 1 Flow chart of patient inclusion and randomization. CCTA, coronary computed tomography angiography; CT-FFR, CT fractional flow reserve; CT-MPI, CT myocardial perfusion imaging; ICA, invasive coronary angiography; OMT, optimal medical treatment.

sequence generation (for example, if time is 19:35:14, the random seed shall be set as 193514). Based on the random seed, a random number table ranging from 0 to 1 will be generated and the allocation of participants will be determined by the size of corresponding random numbers. The random seed and random number table will remain confidential until the end of trial. Sequentially numbered, opaque, sealed envelope (SNOSE) technique will be used to achieve allocation concealment. Randomization group will be written on paper and kept in sealed opaque envelopes labeled with serial numbers. Once the patient is consent to participate, the envelope with corresponding serial number shall be opened and the participant will be assigned according to instructions in envelope.

\section{Scan protocol}

\section{CCTA + dynamic CT-MPI group CCTA + dynamic CT-MPI protocol}

A third generation dual-source CT (SOMATOM Force, Siemens Healthcare, Forchheim, Germany) will be employed for CCTA and dynamic CT-MPI acquisition. CCTA will be firstly performed and on-site evaluation will be performed by a cardiovascular radiologist to interpret CCTA findings. Dynamic CT-MPI will be subsequently performed in the presence of $30 \%$ to $90 \%$ stenosis on any major vessel (diameter $\geq 2 \mathrm{~mm}$ ) as revealed by CCTA. Otherwise, patients will be managed following the study paradigm (Figure 1) without undergoing dynamic CT-MPI. The detailed parameters of CCTA and dynamic CT-MPI acquisition are listed in the Appendix 1.

\section{CCTA and dynamic CT-MPI image reconstruction and analysis}

A medium soft convolution kernel (Bv40) and iterative reconstruction (ADMIRE, level 3) will be used for CCTA reconstruction. The best systolic as well as best diastolic images will be further analyzed using an offline workstation (SyngoVia, version VB20A, Siemens Healthineers, Germany). A stenosis grade of $50 \%$ or greater in an epicardial vessel with diameter $\geq 2 \mathrm{~mm}$ on CCTA will be considered to indicate presence of obstructive $\mathrm{CAD}$.

The CT-MPI images will be reconstructed using a dedicated kernel for reduction of iodine beam-hardening artifacts (Qr36) and analyzed using a CT-MPI software package (VPCT, SyngoVia, version VB20A, Siemens Healthineers, Germany). Quantitative analysis of MBF will 
be measured on a segment base and related details are listed in the Data Supplement. According to the previous study, the absolute $\mathrm{MBF}$ value $<100 \mathrm{~mL} / 100 \mathrm{~mL} / \mathrm{min}$ will be considered as the presence of myocardial ischemia (7).

\section{CCTA + CT-FFR group}

CCTA acquisition settings will be identical to the CCTA protocol used in CCTA + CT-MPI group.

\section{Image reconstruction and analysis}

CCTA reconstruction and image analysis will be as same as aforementioned in the CCTA + dynamic CT-MPI group. Lesion-specific CT-FFR values will be measured for all stenosis with diameter stenosis $\geq 30 \%$ on any epicardial vessel (diameter $\geq 2 \mathrm{~mm}$ ), using a machine learning (ML)based algorithm (cFFR, version 3.0, Siemens Healthineers, Germany) of which the diagnostic performance has been validated against invasive FFR in previous studies $(12,13)$. The detailed measurements of CT-FFR are listed in the Data Supplement. Downstream treatment strategy will be decided based on the results of CT-FFR (Figure 1).

\section{Radiation dose}

Radiation dose of both CT tests and invasive treatment stratum will be recorded. And 0.014 will be used as conversion factor to calculate effective dose.

\section{Downstream treatment strategy}

\section{CCTA + dynamic CT-MPI group}

According to CCTA results, patients without lesions of stenotic extent $\geq 30 \%$ in all major epicardial vessels (diameter $\geq 2 \mathrm{~mm}$ ) will be allocated to optimal medical therapy (OMT) group and patients with at least one lesion of stenosis extent $\geq 90 \%$ will be referred for ICA directly without undergoing dynamic CT-MPI. Patients with at least one $30-90 \%$ stenosis will be further evaluated using dynamic CT-MPI. For patients undergoing dynamic CT-MPI, all participants with lesion-specific absolute $\mathrm{MBF}<100 \mathrm{~mL} / 100 \mathrm{~mL} / \mathrm{min}$ of related territories will be considered as positive and referred for ICA. Further treatment strategy will be independently determined by interventional cardiologists according to the results of ICA and/or invasive FFR [invasive FFR will be performed in necessary cases determined on-site by interventional cardiologists, following the recommendation of Guidelines for the diagnosis and management of chronic coronary syndromes issued by European Society of Cardiology (14)].
In contrast, patients without the presence of myocardial ischemia will be allocated to OMT group.

\section{CCTA + CT-FFR group}

Patients who have at least one lesion with CT- FFR $\leq 0.80$ or stenosis extent $\geq 90 \%$ on any major epicardial vessel (diameter $\geq 2 \mathrm{~mm}$ ) will be referred for ICA and further treatment will be determined by interventional cardiologists according to the results of ICA and/or invasive FFR (if needed). Patients with CT-FFR $>0.80$ in all stenoses will receive OMT.

\section{Follow-up}

Follow-up will be performed at 3rd month, 6th month, 1 year and 2-year after the CT examination. Clinical outcomes, overall radiation exposure and medical cost will be recorded by checking medical records or via outpatient clinic visit.

Clinical outcomes include: (I) rehospitalization due to aggravated angina symptoms (e.g., unstable angina); (II) late revascularization (PCI or CABG performed more than 90 days after baseline CT examination); (III) non-fatal myocardial infarction; (IV) cardiac death (e.g., myocardial infarction, heart failure, fatal arrhythmia); (V) other relevant vascular diseases including cerebrovascular diseases (ischemic stroke, transient ischemic attack, intracerebral hemorrhage, and subarachnoid hemorrhage), pulmonary embolism, ruptured aortic aneurysm, dissecting aneurysm, or other vascular causes.

\section{Study endpoint}

\section{Primary endpoint}

The primary endpoint of the study is the rate of unnecessary ICA within 90 days, and unnecessary ICA is defined as ICA without revascularization.

\section{Secondary endpoints}

The secondary endpoints include: (I) a composite of major adverse cardiac events (MACE, defined as all-cause mortality, non-fatal myocardial infarction, rehospitalization aggravated angina symptoms, and late revascularization); (II) symptom change at 1 year; (III) the rate of late revascularization after CT examination; (IV) reclassification rate of CCTA + CT-MPI and CCTA + CT-FFR guided strategies compared with CCTA alone; (V) overall radiation dose, contrast media usage and medical cost. 


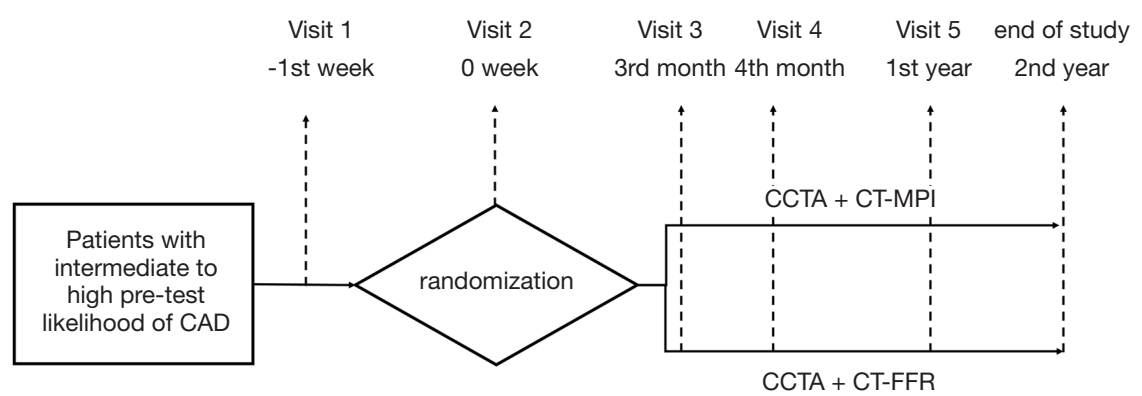

Figure 2 The information of clinical visits. CCTA, coronary computed tomography angiography; CT-FFR, CT fractional flow reserve; CTMPI, CT myocardial perfusion imaging.

\section{Baseline and follow-up visits}

The information of clinical visits is shown in Figure 2.

\section{Patient screening (visit 1)}

All the patients fulfilling inclusive criteria will be invited to attend screening visit. At this visit patients will (I) undergo assessment of CCS; (II) undergo physical examination; (III) be recorded for past medical history, medication history and the results of relevant laboratory tests of the patient during routine medical treatment in the past 6 months.

\section{CT examination (visit 2)}

At this visit patients will (I) undergo CT examination; (II) be recorded the stress heart rate and blood pressure during dynamic CT-MPI examination; (III) be measured for CCTA and dynamic CT-MPI quantitative parameters; (IV) be recorded for adverse reactions (if any).

\section{Follow-up visit (visit 3-5)}

The patients will be followed-up by telephone at the $3 \mathrm{rd}$ month, 6th month and 1 year after the CT examination (visit 3-5) and they will (I) be recorded their ICA results (if subsequent ICA performed), and whether they receive PCI treatment; (II) be followed up for related adverse events; (III) be followed up for any study endpoints; (IV) undergo assessment of clinical symptoms, the method is the same as in visit 1 ; (V) be followed up for medication usage and the relevant laboratory examinations and other examination results (if any).

\section{Last visit}

After the CT examination, the patients will be followed up by telephone in the second year. This visit completes the following tasks: (I) record any study endpoints; (II) record any related adverse events.

\section{Data management}

\section{Data collection}

The study will be conducted in accordance with ICH guidelines (International Council for Harmonisation of Technical Requirements for Pharmaceuticals for Human Use guidelines) and good clinical practice (GCP) standard. Investigators will first collect all original patient information by using a "Medical Record Worksheet", which will be served as "source information" and will be kept and maintained by the investigators. Then, all data specified by the protocol will be reported by the investigators onto a web-based electronic case report forms (CRF) through an electronic data capture (EDC) system. Data query report will be generated by data manager.

\section{Quality control}

Data quality control will include the following steps: (I) edit and logic errors will be checked using EDC system at data entry; (II) data query listing; (III) on-site monitoring in all patients regarding enrollment and consent, completion and documentation of original medical chart and protocol compliance; (IV) remote source document verification (rSDV) in $50 \%$ patients and on-site SDV in $10 \%$ patients for each site; (V) site auditing will be performed in a random fashion to ensure proper study conduct; (VI) personnel in the imaging lab at each site will be trained for qualification prior to enrolling patients.

\section{Statistical analysis}

Statistical analysis will be conducted using commercially 
available statistical software (SPSS Statistical Software version 23.0). Analyses used intention-to-treat populations and were repeated in per- protocol populations. Multiple imputation (by fully conditional specification) will be used for missing data to ensure all participants could be included in the analysis, and avoid treating unknown values as certainly known. The assumption of normal distribution will be checked using One-sample Kolmogorov-Smirnov test. Quantitative variables will be expressed as means \pm standard deviations for normal distribution and median and quartiles for non-normal distribution. Categorical variables will be depicted as a count (\%). Fisher's exact test or the chi-square test will be used to compare categorical variables according to the data cell size. Normally distributed variables will be compared with the student's $t$ test and nonnormally distributed variables will be compared with the Wilcoxon signed-rank test. The Cox proportional hazards regression will be used to calculate hazard ratio with $95 \%$ CI for the between-group comparison of secondary end points, and the log-rank test will be used to calculate the corresponding P. Survival curves are presented graphically using Kaplan-Meier plots. A two-tail probability value of $\mathrm{P}<0.05$ will be considered statistically significant.

\section{Sample size calculation}

Sample size will be estimated using PASS (Power Analysis and Sample Size) software (version 11.0; PASS, NCSS, LLC). According to previous studies, it can be assumed that the event rate would be $35 \%$ in CCTA + CT-FFR guided group and 20\% in CCTA + CT-MPI guided group $(6,15,16)$. Thus, enrollment of 370 subjects $(185$ subjects for CCTA + CT-FFR guided group and 185 subjects for CCTA + dynamic CT-MPI guided group) will be estimated to provide this study $90 \%$ power to detect $15 \%$ different in the frequency of ICA without coronary revascularization at a one-sided 0.025 level of significance. When a drop-out rate of $10 \%$ is assumed, a total enrollment of 412 subjects should be required.

\section{Discussion}

Functional assessment is recommended in patients with intermediate to high pre-test probability $(\geq 15 \%)$ of CAD in order to optimize treatment strategy (11). CT-MPI and CT-FFR are expected to be promising non-invasive techniques to provide functional information of coronary stenoses. Several studies have demonstrated that CTFFR and CT-MPI both have good diagnostic accuracy to detect hemodynamic significance of coronary stenosis with reference to MR MPI or FFR $(2,4,5)$. Furthermore, previous investigations showed that CT-FFR as well as CCTA + CT-MPI strategy both can reduce unnecessary ICA, as compared with CCTA strategy alone $(6,17)$.

More recently, owe to technical development, dynamic CT-MPI using third generation dual source CT has been showed to be superior over ML-based CT-FFR for detection of ischemic stenosis (7) [diagnostic accuracy on vessel-based: $94 \%$ (148 of 157) vs. 78\% (122 of 157), $\mathrm{P}=0.04]$. Despite of the need for additional image acquisition and contrast medium usage, CCTA + dynamic CT-MPI may better rule out functionally significant lesions with higher positive predictive value (7) and consequently avoid unnecessary ICA. However, there is no existing data for direct comparison of these two protocols. Thus, it is of clinical interest to investigate which method has the superiority with regard to reducing unnecessary invasive tests and improving prognosis.

CT-PRECISION study is the first multicenter, prospective, randomized trial to directly compare the clinical values of CCTA + dynamic CT-MPI strategy and CCTA + CT-FFR strategy in terms of optimizing treatment strategy, prognostic discrimination, cost-effectiveness, and radiation exposure. It is expected to provide valuable information on optimal diagnostic strategies to guide proper treatment in patients with intermediate to high pre-test probability of CAD.

\section{Trial registration}

The study is registered at Chinese Clinical Trial Registry (ChiCTR) with the identifier number ChiCTR2000041102 (version 1.0). Two centers will contribute data after the beginning of enrollment, with a planned total sample size of 412 patients. The expected duration for patient enrollment will be 12 months. Follow-up will be finished 24 months after the end of enrollment. Total study duration is expected to be approximately 42 months including data analysis. The first enrollment is planned for January 2021 and enrollment is planned to be finished at January 2022.

Protocol version: 1.0; Data: $1^{\text {st }}$ October 2020.

\section{Acknowledgments}

Funding: This study is supported by Medical Guidance Scientific Research Support Project of Shanghai Science and Technology Commission (Grant No.: 19411965100) and Shanghai Municipal Education Commission- 
Gaofeng Clinical Medicine Grant Support (Grant No.: 20161428).

\section{Footnote}

Reporting Checklist: We present the study in accordance with the SPIRIT reporting checklist. Available at http://dx.doi. org/10.21037/cdt-21-57

Peer Review File: Available at http://dx.doi.org/10.21037/ cdt-21-57

Conflicts of Interest: All authors have completed the ICMJE uniform disclosure form (available at: http://dx.doi. org/10.21037/cdt-21-57). Dr. JZ receives research grant from Siemens Healthineers. The other authors have no conflicts of interest to declare.

Ethical Statement: The authors are accountable for all aspects of the work in ensuring that questions related to the accuracy or integrity of any part of the work are appropriately investigated and resolved. The study will be conducted in accordance with the Declaration of Helsinki (as revised in 2013). Hospital ethic committees of Shanghai General Hospital and Shanghai Jiao Tong University Affiliated Sixth People's Hospital approve this prospective study (approval ID: 2020-173) and all patients will be asked to sign informed consents before enrollment. Any amendments of the protocol will be submitted to hospital ethic committees for review. Detailed clinical characteristics will be recorded.

Open Access Statement: This is an Open Access article distributed in accordance with the Creative Commons Attribution-NonCommercial-NoDerivs 4.0 International License (CC BY-NC-ND 4.0), which permits the noncommercial replication and distribution of the article with the strict proviso that no changes or edits are made and the original work is properly cited (including links to both the formal publication through the relevant DOI and the license). See: https://creativecommons.org/licenses/by-nc-nd/4.0/.

\section{References}

1. Douglas PS, Hoffmann U, Patel MR, et al. PROMISE Investigators. Outcomes of anatomical versus functional testing for coronary artery disease. $\mathrm{N} \mathrm{Engl} \mathrm{J} \mathrm{Med}$ 2015;372:1291-300.
2. Nørgaard BL, Leipsic J, Gaur S et al. NXT Trial Study Group. Diagnostic performance of noninvasive fractional flow reserve derived from coronary computed tomography angiography in suspected coronary artery disease: the NXT trial (Analysis of Coronary Blood Flow Using CT Angiography: Next Steps). J Am Coll Cardiol 2014;63:1145-55.

3. Ihdayhid AR, Norgaard BL, Gaur S et al. Prognostic Value and Risk Continuum of Noninvasive Fractional Flow Reserve Derived from Coronary CT Angiography. Radiology 2019;292:343-51.

4. Bamberg F, Marcus RP, Becker A et al. Dynamic myocardial CT perfusion imaging for evaluation of myocardial ischemia as determined by MR imaging. JACC Cardiovasc Imaging 2014;7:267-77.

5. Coenen A, Rossi A, Lubbers MM et al. Integrating CT Myocardial Perfusion and CT-FFR in the Work-Up of Coronary Artery Disease. JACC Cardiovasc Imaging 2017;10:760-70.

6. Yu M, Shen C, Dai X, et al. Clinical Outcomes of Dynamic Computed Tomography Myocardial Perfusion Imaging Combined With Coronary Computed Tomography Angiography Versus Coronary Computed Tomography Angiography-Guided Strategy. Circ Cardiovasc Imaging 2020;13:e009775.

7. Li Y, Yu M, Dai X, et al. Detection of Hemodynamically Significant Coronary Stenosis: CT Myocardial Perfusion versus Machine Learning CT Fractional Flow Reserve. Radiology 2019;293:305-14.

8. Rossi A, Merkus D, Klotz E, et al. Stress myocardial perfusion: imaging with multidetector CT. Radiology 2014;270:25-46.

9. Min JK, Taylor CA, Achenbach S, et al. Noninvasive Fractional Flow Reserve Derived From Coronary CT Angiography: Clinical Data and Scientific Principles. JACC Cardiovasc Imaging 2015;8:1209-22.

10. Nørgaard BL, Hjort J, Gaur S, et al. Clinical Use of Coronary CTA-Derived FFR for Decision-Making in Stable CAD. JACC Cardiovasc Imaging 2017;10:541-50.

11. Knuuti J, Ballo H, Juarez-Orozco LE, et al. The performance of non-invasive tests to rule-in and ruleout significant coronary artery stenosis in patients with stable angina: a meta-analysis focused on post-test disease probability. Eur Heart J 2018;39:3322-30.

12. Tesche C, De Cecco CN, Baumann S, et al. Coronary CT Angiography-derived Fractional Flow Reserve: Machine Learning Algorithm versus Computational Fluid Dynamics Modeling. Radiology 2018;288:64-72. 
13. Coenen A, Kim YH, Kruk M, et al. Diagnostic Accuracy of a Machine-Learning Approach to Coronary Computed Tomographic Angiography-Based Fractional Flow Reserve: Result From the MACHINE Consortium. Circ Cardiovasc Imaging 2018;11:e007217.

14. Knuuti J, Wijns W, Saraste A, et al 2019 ESC Guidelines for the diagnosis and management of chronic coronary syndromes. Eur Heart J 2020;41:407-77.

15. Pontone G, Baggiano A, Andreini D, et al. Dynamic Stress Computed Tomography Perfusion With a Whole-Heart Coverage Scanner in Addition to Coronary Computed Tomography Angiography and Fractional Flow Reserve Computed Tomography Derived. JACC Cardiovasc

Cite this article as: Dai X, Yu Y, Yu L, Zhang L, Zhang J. Design and rationale of randomized CT-PRECISION study. Cardiovasc Diagn Ther 2021;11(3):760-767. doi: 10.21037/cdt$21-57$
Imaging 2019;12:2460-71.

16. Douglas PS, De Bruyne B, Pontone G, et al. 1-Year Outcomes of FFRCT-Guided Care in Patients With Suspected Coronary Disease: The PLATFORM Study. J Am Coll Cardiol 2016;68:435-45.

17. Douglas PS, Pontone G, Hlatky MA, et al. Clinical outcomes of fractional flow reserve by computed tomographic angiography-guided diagnostic strategies vs. usual care in patients with suspected coronary artery disease: the prospective longitudinal trial of FFR(CT): outcome and resource impacts study. Eur Heart J 2015;36:3359-67. 


\section{CCTA + dynamic CT-MPI group}

Patients will be asked to refrain from intake of $\beta$-blocker or caffeine for 24 hours and to maintain fast for 4 hours before the scan.

\section{CCTA + Dynamic CT-MPI protocol}

A third generation dual-source CT (SOMATOM Force, Siemens Healthcare, Forchheim, Germany) will be employed for CCTA and dynamic CT-MPI acquisition. Calcium score will be firstly performed to calculate the calcification burden of each epicardial vessels. A bolus of contrast media (iopamidol $370 \mathrm{mg}$ iodine/mL, Bayer, Germany) will be injected into the antecubital vein at the rate of $4.5-5 \mathrm{~mL} / \mathrm{s}$, followed by injection of a $40 \mathrm{~mL}$ saline flush, using a dualbarrel power injector (Tyco-Mallinckrodt, US). The amount of contrast media will be determined according to the patient's body weight and the scanning time. Prospective ECG-triggered sequential acquisition will be performed in all participants for CCTA, with triggering window coveting from end-systolic to mid-diastolic phase. The detailed parameters are listed as follow: collimation $=96 \mathrm{~mm} \times 0.6 \mathrm{~mm}$, reconstructed slice thickness $=0.75 \mathrm{~mm}$, reconstructed slice interval $=0.5 \mathrm{~mm}$, rotation time $=250 \mathrm{~ms}$ and application of automated tube voltage and current modulation (CAREKv, CAREDose 4D, Siemens Healthineers, Germany). The reference tube current will be set as $320 \mathrm{mAs}$ and the reference tube voltage will be set as $100 \mathrm{kVp}$.

On-site evaluation will be performed by a cardiovascular radiologist to interpret CCTA findings. Dynamic CTMPI will be subsequently performed if CCTA revealed at least one lesion with stenosis extent from $30 \%$ to $90 \%$ on any major epicardial vessel (diameter $\geq 2 \mathrm{~mm}$ ). Otherwise, patients will be managed following the study paradigm (Figure 1) without undergoing dynamic CT-MPI.

The scan range of dynamic CT-MPI will be planned based on the calcium score images to cover the whole left ventricle. Intravenous ATP infusion will be maintained for 3 minutes at $160 \mu \mathrm{g} / \mathrm{kg} / \mathrm{min}$ before CT-MPI scan. A bolus of contrast media $(50 \mathrm{~mL}$, Iopamidol, $370 \mathrm{mg}$ iodine $/ \mathrm{mL}$, Bayer, Germany) will be injected into antecubital vein at the rate of $6 \mathrm{~mL} / \mathrm{s}$, followed by a $40 \mathrm{~mL}$ saline flush. Dynamic CT-MPI acquisition will be started 4 seconds after the begin of contrast injection. The end-systolic phase (triggered at $250 \mathrm{~ms}$ after the $\mathrm{R}$ wave in all participants) is set for the dynamic acquisition by using a shuttle mode technique with a coverage of $10.5 \mathrm{~cm}$ for complete imaging of the whole left ventricle. Scans will be launched every second or third heart cycle according to participants' heart rate, resulting in a series of 10 to 15 phases acquired over a fixed period of 32 s. The acquisition parameters of dynamic CT-MPI are listed as follow: collimation $=96 \mathrm{~mm} \times 0.6 \mathrm{~mm}$, CARE $\mathrm{kV}$ will be used and the reference tube voltage $=80 \mathrm{kVp}$, rotation time $=250 \mathrm{~ms}$, CARE dose 4D will be used and the effective current $=300 \mathrm{mAs}$, reconstructed slice thickness $=3 \mathrm{~mm}$ and reconstructed slice interval $=2 \mathrm{~mm}$.

\section{CCTA and dynamic CT-MPI image reconstruction and analysis}

CCTA images will be reconstructed using a medium soft convolution kernel (Bv40) and iterative reconstruction (ADMIRE, level 3). The best systolic as well as best diastolic images will be transferred to an offline workstation (SyngoVia, version VB20A, Siemens Healthineers, Germany) for further analyses.

The diameter stenosis will be calculated as (reference diameter - minimal lumen diameter)/reference diameter and will be measured manually with a digital caliper at the narrowest level of the lesion and the proximal reference on the cross-sectional images. A stenosis grade of $50 \%$ or greater in an epicardial vessel with diameter $\geq 2 \mathrm{~mm}$ on CCTA will be considered to indicate presence of obstructive CAD.

The CT-MPI images will be reconstructed using a dedicated kernel for reduction of iodine beam-hardening artifacts (Qr36) and analyzed using a CT-MPI software package (VPCT, SyngoVia, version VB20A, Siemens Healthineers, Germany). Motion correction will be applied in necessary cases to correct for breathing-related mis-registration of the left ventricle. For quantification of myocardial blood flow (MBF), the influx of contrast medium will be measured using the arterial input function (AIF). The AIF will be sampled in the descending aorta by including both the cranial and caudal sections. For quantification of the MBF, the myocardial time attenuation curves will be coupled with the AIF using a hybrid deconvolution model. For quantitative analysis, the region of interest (ROI) will be drawn to cover the whole area of suspected perfusion defect within the segment or cover the whole segment when perfusion defect will be absent. The stenosis-subtended territories and reference territories will be each determined according to the fusion images of coronary vasculature and perfusion map. The mean value of stress MBF will be measured for each segment of both stenosis-subtended territories and reference territories. 
According to the previous study, the absolute $\mathrm{MBF}$ value $<100 \mathrm{~mL} / 100 \mathrm{~mL} / \mathrm{min}$ will be considered as the presence of myocardial ischemia (7).

\section{CCTA + CT-FFR group}

CCTA acquisition settings will be identical to the CCTA protocol used in CCTA + CT-MPI group.

\section{Image reconstruction and analysis}

CCTA images will be reconstructed using a medium soft convolution kernel (Bv40) and iterative reconstruction (ADMIRE, level 3). The best systolic as well as best diastolic images will be transferred to an offline workstation (SyngoVia, version VB20A, Siemens Healthineers, Germany) for further analyses.

Lesion-specific CT-FFR values will be measured for all stenosis with diameter stenosis $\geq 30 \%$ on any epicardial vessel with diameter $\geq 2 \mathrm{~mm}$. The current study employs a machine learning (ML)-based approach for CT-FFR simulation (cFFR, version 3.0, Siemens Healthineers, Germany). This model will be trained on a large database of synthesized coronary anatomies, where the reference values are computed by using a computational fluid dynamics based model. For the on-site processing, after CCTA data is successfully loaded, the centerline and luminal contours for whole coronary tree are automatically generated. The centerline and luminal contour are fundamental and critical information for computing CT-FFR value. They are manually adjusted when needed. Users then manually identify all stenotic lesions to extract their geometrical features required for cFFR algorithm. Finally, those data are input into the pre-learned model and cFFR is computed automatically at all locations in the coronary arterial tree, and the resulting values are visualized by color-coded $3 \mathrm{D}$ coronary maps. The lesion-specific CT-FFR values are measured at the distal shoulder of the lesion, where no plaque can be detected. Downstream treatment strategy will be decided based on the results of CT-FFR (Figure 1). 